\title{
Aspectos epidemiológicos da dengue no Estado de Mato Grosso, Brasil: série
}

\section{temporal 2001-2018}

\author{
Epidemiological aspects of dengue in the State of Mato Grosso, Brazil: time series between the \\ years 2001 and 2018 \\ Aspectos epidemiológicos del dengue en el Estado de Mato Grosso, Brasil: series de tiempo entre los
}

años 2001 y 2018

Tatiane Gomes de Almeida

ORCID: https://orcid.org/0000-0001-9406-9831 Universidade do Estado de Mato Grosso, Brasil

E-mail: tatiane.almeida@unemat.br

Arno Rieder

ORCID: https://orcid.org/0000-0002-9027-2549 Universidade do Estado de Mato Grosso, Brasil E-mail: riederarno@gmail.com

Ernandes Sobreira Oliveira Junior ORCID: https://orcid.org/0000-0002-6953-6917 Universidade do Estado de Mato Grosso, Brasil E-mail: ernandes.sobreira@gmail.com

Claumir Cesar Muniz

ORCID: https://orcid.org/0000-0002-2082-2234 Universidade do Estado de Mato Grosso, Brasil E-mail: claumir@unemat.br

Alexandra Oliveira Ramos

ORCID: https://orcid.org/0000-0001-6064-9045 Universidade do Estado de Mato Grosso, Brasil E-mail: ale_oliveiracac@hotmail.com

Patrícia Alves Pereira

ORCID: https://orcid.org/0000-0002-8642-2102 Universidade do Estado de Mato Grosso, Brasil E-mail: patriciaenfpeda@hotmail.com

\begin{abstract}
Resumo
Objetivo: Descrever as características epidemiológicas da dengue no estado de Mato Grosso, Brasil, 2001 a 2018.Métodos: Dados obtidos no Departamento de Informática do Sistema Único de Saúde (DATASUS) para análises da letalidade, incidência e internação hospitalar.Resultados: No Estado de Mato Grosso foram 292.756 notificações e 256 óbitos no período estudado. O pico de incidência e letalidade foi em 2009 (masculino 1544,1; feminino 1960,3; masculino 268,1; feminino 107,2 casos/100 mil habitantes), respectivamente. Porém, o ápice na taxa de internação hospital (masculino 156,4/100 mil habitantes; feminino 178,1/100 mil habitantes) foi em 2010. O pico da taxa de incidência nas faixas etárias foi em 2009, em que crianças $(<1$ ano) apresentaram maior taxa de incidência, sendo 2156,8/100 mil habitantes neste ano. Depois os adolescentes (10 a 19 anos) com 2003,4/100 mil habitantes); os adultos (20 a 59 anos; 1740,4/100 mil habitantes); as crianças (1 a 9 anos; 1653,8/100 mil habitantes) e as menores taxas foram nos idosos (>60 anos; 1257,1/100 mil habitantes). A maior taxa de letalidade foi nos idosos $(435,9 / 100$ mil habitantes), depois nas crianças < de 1 ano (335,2/100 mil habitantes); nas crianças de 1 a 9 anos (86,9/100 mil habitantes); nos adultos (54/100 mil habitantes) e; o de menor taxa foi nos adolescente com 40,3/100 mil habitantes. Conclusão: A dengue revelou picos epidêmicos com altas taxas de incidência. $\mathrm{O}$ sexo feminino adoece e hospitaliza mais que o masculino, porém o masculino tem maior letalidade. Os adultos adoecem mais, sendo a letalidade maior nos idosos.
\end{abstract}

Palavras-chave: Epidemiologia; Letalidade; Incidência; Internação hospitalar.

\section{Abstract}

Objective: To describe the epidemiological characteristics of dengue in the State of Mato Grosso, Brazil, from 2001 to 2018.Methods: Data were obtained from the Informatics Department of the Unified Health System (DATASUS) for analyzes of lethality, incidence and hospitalization. Results: In the state of Mato Grosso there were 292,756 notifications and 256 deaths in the period studied. The peak of incidence and lethality was in 2009 (male 1544.1; 
female 1960.3; male 268.1; female 107.2 cases / 100 thousand inhabitants), respectively. However, the hospital admission rate (male 156.4 / 100 thousand inhabitants; female 178.1 / 100 thousand inhabitants) was in 2010. The peak incidence rate in the age groups was in 2009, in which children $(<1$ year $)$ had a higher incidence rate, with 2156.8 / 100 thousand inhabitants this year. Then the teenagers (10 to 19 years old) with $2003.4 / 100$ thousand inhabitants); adults (20 to 59 years old; 1740.4 / 100 thousand inhabitants); children (1 to 9 years; $1653.8 / 100$ thousand inhabitants) and the lowest rates were among the elderly (> 60 years; $1257.1 / 100$ thousand inhabitants). The highest lethality rate was in the elderly 435.9 / 100 thousand inhabitants, then in children $(<1$ year; 335.2 / 100 thousand inhabitants); in children (1 to 9 years; 86.9 / 100 thousand inhabitants); in adults 54/100 thousand inhabitants and; the lowest among adolescents with 40.3 / 100 thousand inhabitants. Conclusion: Dengue showed epidemic peaks with high incidence rates. The female sex gets sick and hospitalizes more than the male, but the male has a higher lethality. Adults become more ill, however, lethality is higher in the elderly.

Keywords: Epidemiology; Lethality; Incidence; Hospital internment.

\section{Resumen}

Objetivo: Describir las características epidemiológicas del dengue en el Estado de Mato Grosso, Brasil, de 2001 a 2018.Métodos: Datos obtenidos del Departamento de Informática del Sistema Único de Salud (DATASUS) para análisis de letalidad, incidencia y hospitalización. Resultados: En el Estado de Mato Grosso se registraron 292.756 notificaciones y 256 defunciones en el período estudiado. El pico de incidencia y letalidad fue en 2009 (hombres 1544,1; mujeres 1960,3; hombres 268,1; mujeres 107,2 casos / 100 mil habitantes), respectivamente. Sin embargo, la tasa de ingreso hospitalario (hombres 156,4 / 100 mil habitantes; mujeres 178,1 / 100 mil habitantes) fue en 2010. La tasa de incidencia máxima en los grupos de edad fue en 2009, en los cuales los niños ( $<1$ año) tuvieron la mayor tasa de incidencia. , con 2156,8 / 100 mil habitantes este año. Luego los adolescentes (10 a 19 años) con 2003,4 / 100 mil habitantes); adultos (20 a 59 años; 1740,4 / 100 mil habitantes); niños (1 a 9 años; 1653,8 / 100 mil habitantes) y las tasas más bajas se dieron entre los ancianos (> 60 años; 1257,1 / 100 mil habitantes). La mayor letalidad se registró en ancianos 435,9 / 100 mil habitantes, luego en niños (<1 año; 335,2 / 100 mil habitantes); en niños (1 a 9 años; 86,9 / 100 mil habitantes); en adultos 54/100 mil habitantes y; el más bajo entre los adolescentes con 40,3 / 100 mil habitantes. Conclusión: El dengue presentó picos epidémicos con altas tasas de incidencia. El sexo femenino se enferma y hospitaliza más que el masculino, pero el masculino tiene una mayor letalidad. Los adultos se enferman más, sin embargo, la letalidad es mayor en los ancianos.

Palabras clave: Epidemiologia; Letalidad; Incidencia; Internación hospitalaria.

\section{Introdução}

A dengue, uma doença infeciosa viral, tem como agente patológico um vírus (DENV; Flaviviridae; Flavivírus) que é transmitido por mosquitos fêmeas do Aedes aegypti (Linnaeus, 1762) (Diptera: Culicidae) que também pode transmitir o agente patogênico que causa a chikungunya, zika vírus e febre amarela urbana, sendo que ainda existe um vetor secundário do vírus da dengue, o Aedes albopictus (Ministério da saúde, (2001) e a Organização Mundial da Saúde, (2019)). Além disso, existem quatro principais sorotipos: DENV-1, DENV-2, DENV-3 e DENV-4, no qual a infecção por um deles confere proteção permanente para o mesmo sorotipo (Fogaça, 2017). O período médio de incubação do vírus, após a picada do mosquito fêmea, é de 4 a 7 dias, se replicando dentro de células do sistema mononuclear fagocitário (Marchiore et al., 2020).

Conforme Villar e Gonçalves (2016), o vetor da dengue é um artrópode da família Culicidae, denominado Aedes Aegypti por ter sido identificado primeiramente no Egito, tem a hipótese de sua inserção no continente americano de embarcações oriundas da Europa, durante as primeiras explorações e colonizações européias. Segundo a OMS (2019), a dengue é um problema de saúde pública, e Vilar e Gonçalvez (2016) trazem que o mosquito transmissor do vírus é detectado em todo o mundo e se adapta com facilidade em ambientes com maior aglomeração de pessoas e se prolifera depositando seus ovos em locais com água parada, alimentando-se de sengue durante o início da manhã e antes do anoitecer.

Segundo Viana e Ignotti (2013), a temperatura, umidade relativa do ar e pluviosidade influenciam a dinâmica desse vetor, pois a ocorrência está associada à elevação dos índices pluviométricos e às variações de temperatura. Relatam ainda que, nos meses mais frios e secos há uma redução da densidade vetorial, porém ainda há a reprodução desse mosquito. Nas últimas décadas, a incidência da dengue tem crescido drasticamente em todo o mundo, em que estimativas recentes indicam 390 milhões de infecções de dengue por ano (Organização Pan-Americana de Saúde, 2019). Nas Américas, conforme PHO (2017), 
em 2016, 64,5\% das notificações de dengue ocorreram no Brasil. Essa doença é um grave problema de saúde pública, que acomete e leva a óbito milhares de pessoas anualmente no Brasil. Por essa razão é de interesse para a saúde pública controlar a proliferação do vetor que causa essa doença. Dessa forma, é importante se conhecer o perfil epidemiológico e o comportamento da dengue no Brasil, pois no Estado de Mato Grosso é a doença vetorial de maior incidência (Secretaria Estadual de Saúde do Estado de Mato Grosso, 2019). Diante da importância dessa enfermidade, apresenta-se um estudo de caracterização epidemiológica da dengue no Estado de Mato Grosso em um período de 18 anos (2001 a 2018).

\section{Metodologia}

A pesquisa utilizou dados sobre a dengue no Estado de Mato Grosso correspondente ao período entre 2001 e 2018. O estado é o terceiro maior do Brasil, com uma área de 903.207,019 km2 e área urbana de 519,7 km2, além de possuir 141 municípios, sendo Cuiabá a capital (Instituto Brasileiro de Geografia Estatística (IBGE, 2020)). A temperatura média que gira em torno de $24^{\circ} \mathrm{C}$, podendo a máxima ultrapassar os $40^{\circ} \mathrm{C}$. O clima mais prevalente é o tropical, com chuvas de verão e inverno seco, apresentando período chuvoso, que vai de outubro a abril, e período seco de maio a setembro. O estado ainda apresenta três dos principais biomas do país: Amazônia, Pantanal e Cerrado (Governo do Estado de Mato Grosso, 2020).

Este é um estudo epidemiológico descritivo das taxas de incidência, letalidade e internação hospitalar da dengue no estado de Mato Grosso, no período de 2001 (ano disponível que continha os dados necessários para as análises epidemiológicas) a 2018 (último ano com dados disponíveis). Os dados para o cálculo da incidência, letalidade, e internação hospitalar foram apresentados por sexo e faixa etária.

Os dados referentes à dengue foram coletados no banco de dados eletrônico do Departamento de Informática do Sistema Único de Saúde (DATASUS: http://www2.datasus.gov.br/DATASUS/index.php), do Ministério da Saúde. Utilizou-se os casos notificados disponibilizados no Sistema de Informação de Agravos de Notificação - SINAN; óbitos no Sistema de Informações sobre Mortalidade - SIM e; internação hospitalar no Sistema de Informações Hospitalares do SUS (SIH/SUS).

Esses indicadores epidemiológicos foram utilizados para caracterizar a dengue entre o sexo (masculino e feminino, categorização disponível no DATASUS) e por faixa etária segundo a OMS e disponibilização no site eletrônico do DATASUS (crianças': < de 1 ano; crianças: 1 a 9 anos; adolescentes: 10 a 19 anos; adultos: 20 a 59 anos; idoso: $\geq 60$ anos).

Os dados da população residente no Estado de Mato Grosso, empregadas como denominadores para o cálculo do coeficiente de incidência, letalidade e internação hospitalar da doença foi gerada pelo aplicativo TABNET, desenvolvido pelo DATASUS, sendo que para as análises foram utilizados recursos do programa Tabwin versão 4.15. Para o cálculo da taxa de incidência por sexo e faixa etária, foi considerada como numerador casos novos notificados da doença, e como denominadores, a população informada pela Fundação Instituto Brasileira de Geografia e Estatística (IBGE), que está disponibilizada no Departamento de Informática do Sistema Único de Saúde (DATASUS), multiplicado por 100 mil; para a incidência, como numerador, o número de casos novos notificados e denominador, a população do ano, multiplicado por 100 mil; para a letalidade, como numerador, o número de óbitos do ano e denominador, os casos de dengue no ano, multiplicado por 100 mil; e para a taxa de internação hospitalar como numerador, as internações no ano e denominador, a população do ano, vezes 100 mil. Para a classificação das situações epidêmicas foram utilizados os parâmetros estabelecido pelo Programa Nacional de Controle da Dengue do Ministério da Saúde (2006), sendo valores <100/100 mil habitantes como surto e baixa incidência, entre 100 e 300 casos por 100 mil habitantes como surto epidêmico e média incidência e; valores >300 casos/100 mil habitantes como surtos epidêmicos e alta incidência.

O estudo foi dispensado de apreciação por Comitê de Ética em Pesquisa, uma vez que utilizou exclusivamente dados de acesso público disponibilizado e de livre acesso através da internet no site do DATASUS. Dessa forma, está de acordo com 
os preceitos éticos, não gerando quaisquer danos e identificações de pessoas.

\section{Resultados}

Nos 18 anos (2001-2018) de notificações foram 292.633 casos registrados no Estado de Mato Grosso. Houve um aumento de 731,10\% entre 2001 (2.594) para 2018 (7.312), em que o pico de notificações registradas ocorreu no ano de 2009 (52.477). As notificações apresentaram um comportamento oscilatório, em que os valores mínimos se repetem em subperíodos de quatro ou cinco anos, enquanto que os valores máximos se reproduzem em subperíodos de três e cinco anos. No período considerado houve 256 óbitos, com as maiores frequências nos anos de 2009 (58 mortes) e 2010 (66 mortes). O incremento de óbitos de 2001 (três mortes) para 2018 (oito) foi de 166,66\%.

\subsection{Indicadores epidemiológicos da dengue de 2001 a 2018 entre sexo}

No período analisado foram registrados no DATAUS 292.633 notificações de casos de dengue, sendo 132.567 do sexo masculino, 160.066 do sexo feminino e 62 casos ignorados. Dessas notificações, vieram a óbito 256 pessoas, sendo 154 do sexo masculino e 102 do sexo feminino. $\mathrm{O}$ ano de 2009 teve mais notificações (52.477 casos): 23.626 do sexo masculino e 28.851 do sexo feminino. $\mathrm{O}$ ano de 2010 apresentou maior mortalidade (66 óbitos): 45 do sexo masculino e 21 do sexo feminino e; a menor ocorreu em 2005 (um óbito): sexo feminino nenhuma morte e no sexo masculino apenas um óbito.

\subsubsection{Taxa de incidência por sexo}

A Figura 1 expressa as taxas de incidência entre o sexo masculino e feminino no período analisado (2001-2018) no Estado de Mato Grosso. Durante os 18 anos analisados, tanto o sexo masculino quanto o feminino apresentaram comportamento oscilatório e taxas semelhantes, ou seja, em ambos os sexos a taxa de incidência aumentava ou diminuía no decorrer dos anos, porém sem haver diferenciação significante (ns; $\mathrm{p}>0,05$ ). $\mathrm{O}$ ano que apresentou maior taxa de incidência (casos/100 mil habitantes), no total, foi em 2009 (1748,22). Entretanto, neste ano, para o sexo masculino (1544,18), a taxa esteve abaixo a do feminino (1960,36). O ano de menor incidência, no total, foi em 2004 (89,02). Para o sexo masculino $(80,66)$ e feminino $(97,37)$, as menores taxas também se manifestaram em 2004 (Figura 1). No período de 18 anos a taxa média de incidência foi de 474,1/100 mil habitantes para o sexo masculino e 589,5/100 mil habitantes para o feminino, Entretanto, estatisticamente, a taxa média de incidência $(531,84)$ no período analisado é considerada similar entre os sexos (p > 0,05). 
Figura 1- Taxa de incidência (casos/100 mil habitantes) da dengue por sexo no Mato Grosso, Brasil, 2001-2018.

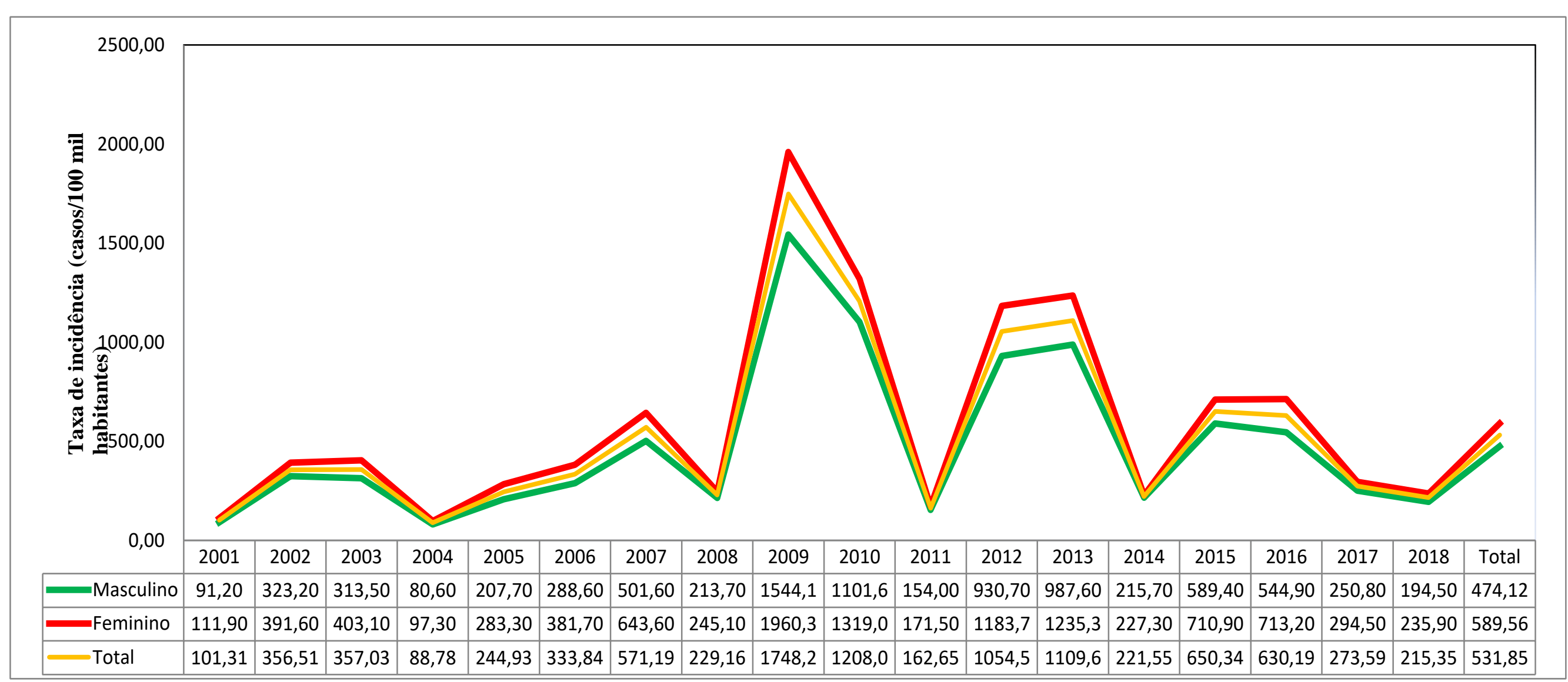

Período (anos)

Fonte: figura elaborada pelos autores, com base de dados do DATASUS (2021). 
Research, Society and Development, v. 10, n. 6, e32610615716, 2021

(CC BY 4.0) | ISSN 2525-3409 | DOI: http://dx.doi.org/10.33448/rsd-v10i6.15716

\subsubsection{Letalidade por sexo}

Durante o período de 18 anos (2001-2018) houve variação das taxas de letalidade entre anos, seja para sexo e no geral (Figura 2). Vieram a a óbitos 256 pessoas, sendo 154 do sexo masculino e 102 do sexo feminino (Figura2).

Figura 2- Taxa de letalidade da dengue por sexo, Mato Grosso, Brasil, 2001-2018.

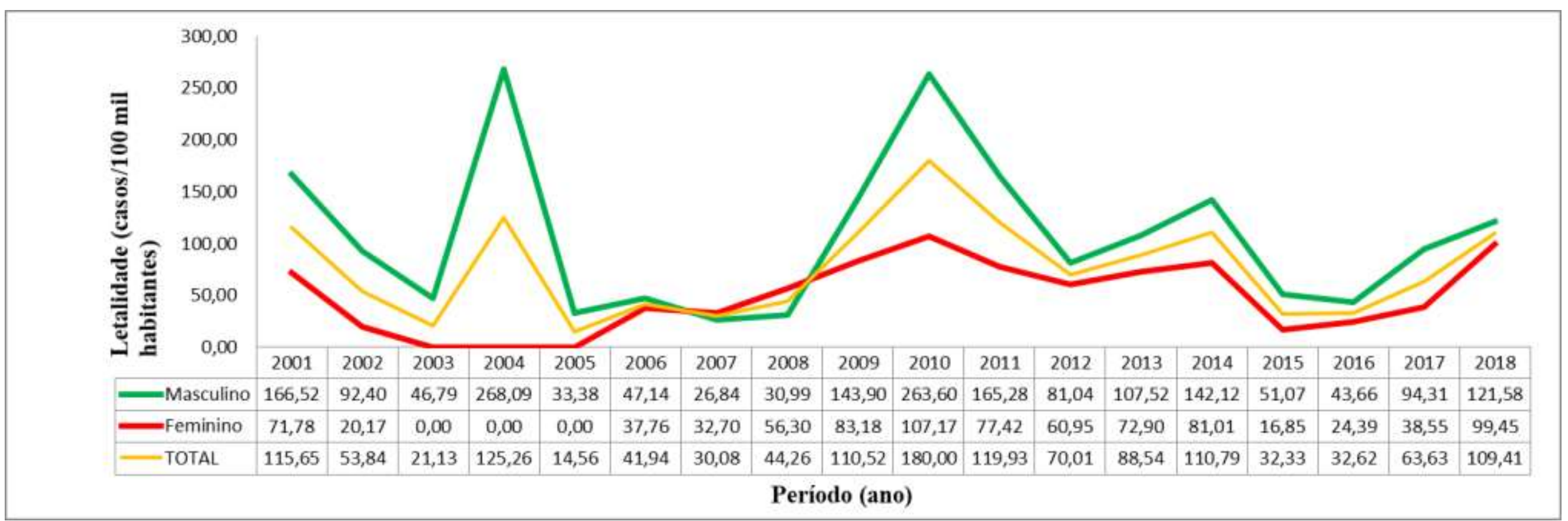

Fonte: figura elaborada pelos autores, com base de dados do DATASUS (2021). 
Durante os 18 anos analisados, o sexo masculino apresentou em números absolutos mais casos de óbitos. A taxa de letalidade por dengue (casos/100 mil habitantes) nos 18 anos estudados, no geral, foi de 87,4; enquanto no sexo masculino foi maior (116,1) e no sexo feminino foi menor (63,7). Nos anos de 2003, 2004 e 2005 o sexo feminino não apresentou letalidade enquanto que o sexo masculino apresentou nesses mesmos anos taxas que oscilaram bastante $(46,8 ; 268,1 ; 33,4$, respectivamente). O pico máximo da taxa de letalidade no período estudado para o sexo masculino foi de 263,6 em 2010. Já para o sexo feminino, no mesmo ano, foi de 107,2. Durante todo o período analisado, percebe-se que a taxa de letalidade foi maior para as pessoas do sexo masculino.

\subsubsection{Internação hospitalar por dengue de 2001 a 2018 por sexo}

No período analisado as taxas de internação hospitalar (casos/100 mil habitantes) apresentaram 25.617 internações, sendo 12.566 do sexo masculinos e 13.051 femininos. Desde o ano de 2001, o maior surto de casos de internação hospitalar por dengue no Estado de Mato Grosso no sexo masculino ocorreu em 2010 (143,4/100 mil habitantes) e 2010 (156,4/100 mil habitantes), e número de internações de 2.223 e 2.424 casos, respectivamente; já para o sexo feminino também foi nesses mesmos anos, 158/100 mil habitantes e 178,3/100 mil habitantes, e número de internações de 2.326 e 2.650 casos, respectivamente. As menores taxas para o sexo masculino ficaram registradas em 2001 (12,3/100 mil habitantes) e feminino em 2017 (9.9/100 mil habitantes). Percebe-se que as taxas de internações entre os sexos oscilaram no decorrer dos anos (Figura 2), porém o sexo masculino apresentou taxa menor (46/100 mil habitantes) no período de estudo, enquanto que o feminino foi maior (49/100 mil habitantes). As internações hospitalares no sexo masculino correspondem a $49 \%$ e o sexo feminino $51 \%$ do total de 25.617 internações. 
Figura-3 Taxa de internação hospitalar, por sexo, Mato Grosso, Brasil, 2001 a 2018.

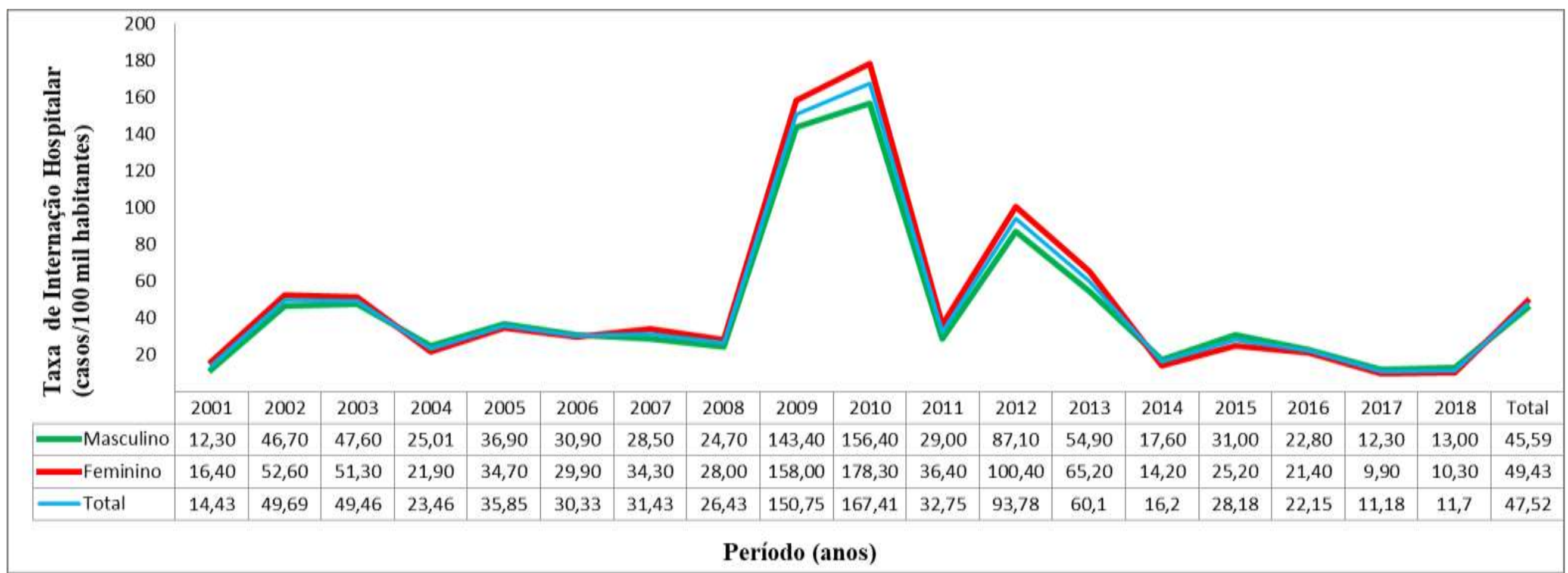

Fonte: figura elaborada pelos autores, com base de dados do DATASUS (2021).

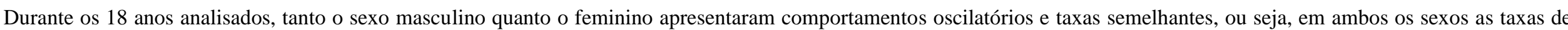

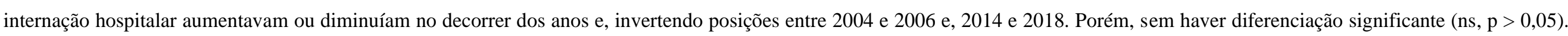

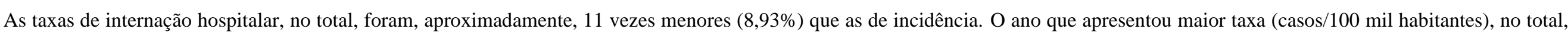

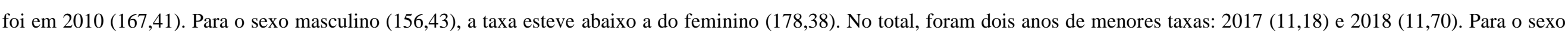
masculino foi em 2017 (12,36) e feminino (10,00). A taxa média de internação hospitalar $(47,52)$ no período analisado foi similar entre os sexos (Figura 3$)$. 
3.2 Indicadores epidemiológicos da dengue de 2001 a 2018 entre faixa etária

3.2.1 Incidência nas faixas etárias

A Figura 4 expressa as taxas de incidência entre as faixas etárias no período analisado (2001-2018) no Estado de Mato Grosso.

Figura 4- Taxa de incidência da dengue por faixa etária, Mato Grosso, Brasil, 2001-2018.

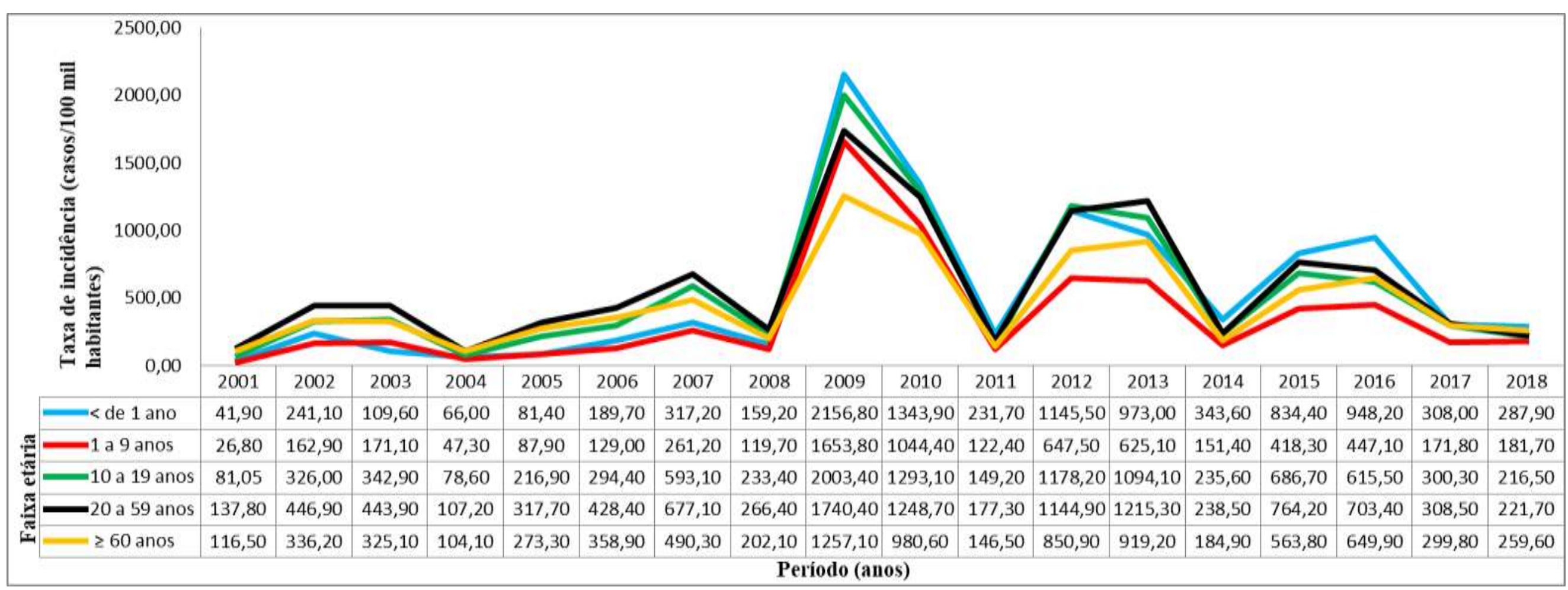

Fonte: figura elaborada pelos autores, com base de dados do DATASUS (2021). 
A faixa etária que mais adoeceu por dengue no período estudado foram os adultos (20 a 59 anos) com uma taxa de incidência de 604,9/100 mil habitantes, correspondendo a 180.547 mil casos notificados. Em segundo lugar foram os adolescentes (10 a 19 anos) com taxa de 546, 5/100 mil habitantes, sendo 57.055 casos notificados. Em terceiro lugar as crianças $<1$ ano com uma taxa de 538,5/100 mil habitantes, sendo 5.071 casos notificados. Em quarto lugar os idosos (> 60 anos) com uma taxa de 489,5/100 mil habitantes, sendo 18,811 casos notificados. E em quinto lugar as crianças (1 a 10 anos) com uma taxa de 352,9/100 mil habitantes, sendo 31.055 casos notificados (Figura 4).

As maiores taxas de incidência ocorreram no ano de 2009. Naquele ano, as crianças $<1$ ano apresentaram maior taxa de incidência entre as demais faixas etárias, sendo 2156,8/100 mil habitantes neste ano (2009). Em segundo lugar os adolescentes (10 a 19 anos) com 2003,4/100 mil habitantes. Em terceiro lugar os adultos (20 a 59 anos) com uma taxa de 1740,4/100 mil habitantes. Em quarto lugar as crianças de 1 a 9 anos com 1653,8/100 mil habitantes. Em último lugar os idosos (> 60 anos) com taxa de morbidade de 1257,1/100 mil habitantes.

\subsubsection{Letalidade nas faixas etárias}

No período estudado, a maior taxa de letalidade foi nos idosos ( > 60 anos) 435,9/100 mil habitantes, depois nas crianças < de 1 ano com 335,2/100 mil habitantes; nas crianças de1 a 9 anos com 86,9/100 mil habitantes; nos adultos (20 a 59 anos) 54/100 mil habitantes; e a menor taxa foi nos adolescentes com 40,3/100 mil habitantes. Nos menores de um ano, nos anos de 2001 a 2007, 2011, 2015, 2016 e 2018 a taxa de letalidade foi 0, porém no ano de 2008 apresentou taxa de 1162,3/100 mil habitantes, sendo essa a maior taxa entre as faixas etárias durante os 18 anos de estudo.

Nas crianças 1 a 9 anos, nos últimos 4 anos de estudos $(2015,2016$, 2017, 2018), a taxa de letalidade foi 0, sendo a maior taxa (212,7/100 mil habitantes) no ano de 2010. Nos adolescentes (10 a 19 anos), nos três primeiros anos (2001, 2001, 2003) a taxa de letalidade foi 0 , sendo em 2004 a maior taxa (215,5/100 mil habitantes). Nos adultos, diferente das outras faixas etárias, todos os anos apresentaram taxa de letalidade não nula, sendo a menor em 2016 (15/100mil habitantes) e a maior em 2010 (116/100 mil habitantes). Nos idosos, nos anos de 2005, 2008 e 2017 a taxa de letalidade foi 0, porém, no ano de 2010 houve a segunda maior taxa (1126,2/100 mil habitantes) de todas as faixas etárias, nos 18 anos analisados. 
3.2.3 Internação hospitalar por dengue de 2001 a 2018 nas faixas etárias

A Figura 5 expressa as taxas de internação hospitalar entre as faixas etárias no período de 2001 a 2019 e, óbitos decorrentes da dengue.

Figura 5- Internação hospitalar e óbitos de dengue por faixa etária, Mato Grosso, Brasil, 2001-2018.

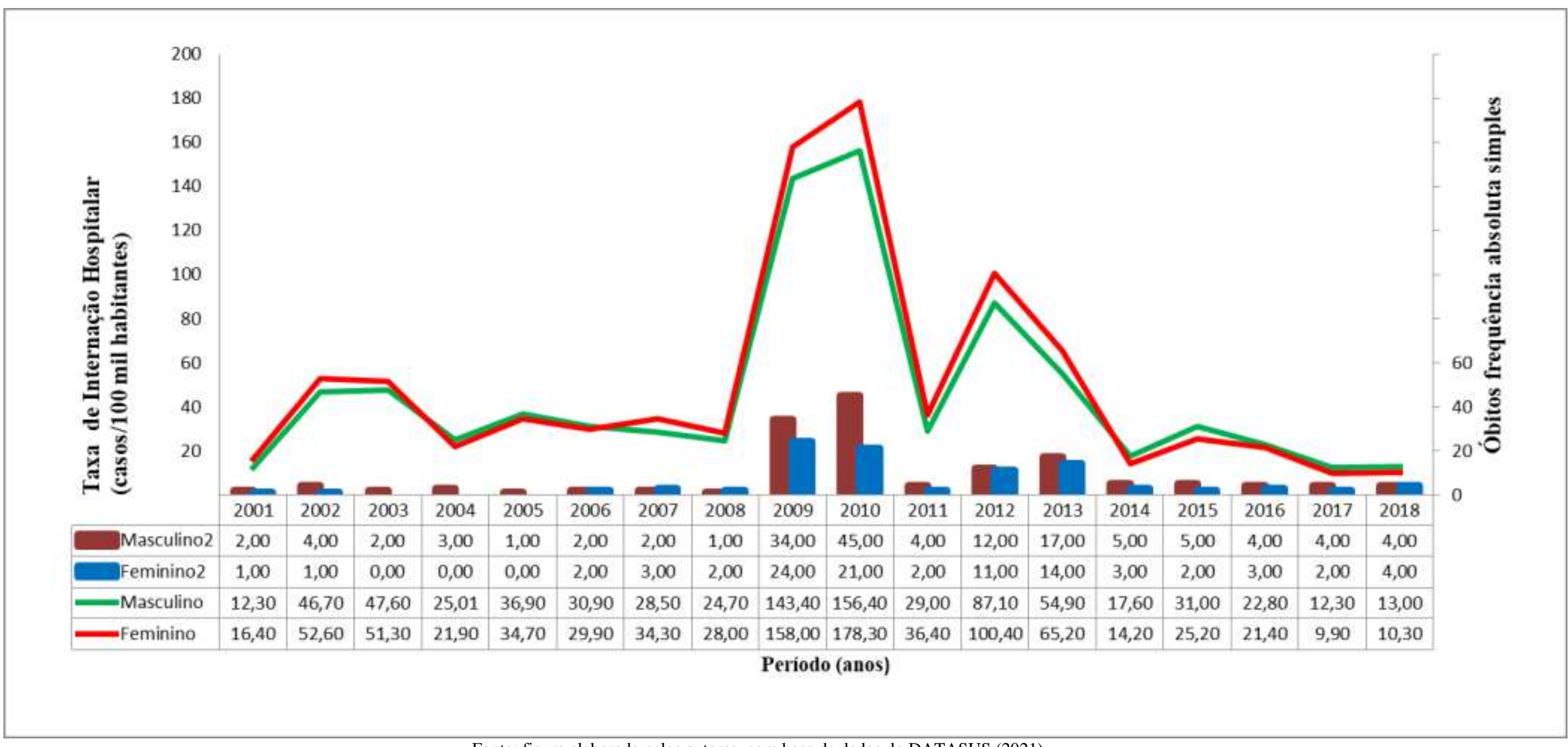

Fonte: figura elaborada pelos autores, com base de dados do DATASUS (2021). 
Research, Society and Development, v. 10, n. 6, e32610615716, 2021

(CC BY 4.0) | ISSN 2525-3409 | DOI: http://dx.doi.org/10.33448/rsd-v10i6.15716

Nas faixas etárias, no período estudado, a taxa média de internação no Estado de Mato Grosso por dengue foi de 47,2 casos /100 mil habitantes, distribuída conforme segue: em crianças menores de um ano com 47,0/100 mil habitantes, crianças de1 a 9 anos com 38,8/100 mil habitantes, adolescentes (10 a 19 anos) com 47,8/100 mil habitantes, adultos (20 a 59 anos) com 47,0/100 mil habitantes e idosos (>=60 anos) com70,1/100 mil habitantes. Percebe-se que os idosos estiveram mais propícios as internações durantes os 18 anos analisados. O ano que teve o pico de maiores internações foi em 2009 e 2010 , com 151,4/100 mil habitantes e 167,1/100 mil habitantes, respectivamente. Os de menores taxas foram nos dois últimos anos, 2017 e 2018, ambos com 11/100mil habitantes. Já o pico de internações na faixa etária nos menores de um ano foi em 2011 (226/100 mil habitantes), na faixa etária das crianças foi em 2009 (203/100 mil habitantes), nos adolescentes foi em 2010 (189/100 mil habitantes), nos adultos também em 2010 (145,8/100 mil habitantes) e nos idosos em 2010 (194,4/100 mil habitantes).

\section{Discussão}

O Brasil vem sofrendo com epidemias de dengue, pois as notificações de casos de dengue mais que dobraram em janeiro de 2019 (21.992 casos), quando comparado com janeiro de 2018 (54.777 casos), um aumento de 149\%. A região Centro-Oeste está em segundo lugar entre as regiões brasileiras que mais registraram casos de dengue (10.827 casos) (Brasil, 2019). O Estado de Mato Grosso apresenta ambiente e clima favorável para a proliferação do vetor transmissor do agente patogênico que causa a dengue, sendo essa doença típica de áreas tropicais e subtropicais, em que a maioria dos casos são registradas nas regiões periféricas e em municípios onde não há planejamento urbano e saneamento básico (De Oliveira et al., 2014).

Dentre as Regiões do Brasil, no período de 2002 a 2012, o Centro Oeste apresenta uma taxa alta de incidência de dengue (Bohm et al., 2016). Desde o ano de 2001, o maior surto de dengue no Estado de Mato Grosso ocorreu em 2009 (52.477 casos), esse mesmo cenário também ocorreu no Estado de Rondônia neste mesmo ano (15.378 casos) sendo o período analisado de 1999 a 2010 (Lucena et al., 2011). No período analisado (2001 a 2018) as taxas de incidência foram maiores no sexo feminino, isso se observou também no estudo realizado em Fortaleza-Ceará, no período de 2001 a 2012, sendo a variação da taxa de incidência entre 662,0 casos/100 mil habitantes em 2006 e 1.675,5 casos/100 mil habitantes em 2012 para o sexo feminino (Oliveira et al., 2018), e também no Estado de Goiás no período de 2016-2018, em que o sexo feminino apresentou $54,8 \%$ dos casos (De Lima Oliveira et al., 2020). Porém, nas taxas de letalidade o comportamento é diferente, uma vez que as maiores taxas foram registradas no sexo masculino, cujo comportamento se repete em Goiás.

Sendo assim, o sexo feminino adoece mais, todavia a dengue é mais letal no sexo masculino. Isso também ocorreu em um estudo realizado no Estado da Bahia no período de 2007 a 2017, teve 58\% das taxas de incidência registradas no sexo feminino, cujo achado também foi evidenciado por outros estudos, em que uma provável explicação a permanência por mais tempo das mulheres em seus domicílios, sendo esse o hábitat favorito do Aedes aegypti (Martins et al., 2015). Segundo o relatório World Health Statistics (2019), há uma redução da expectativa de vida mais em homens do que em mulheres e, em 2016 a probabilidade de uma pessoa morrer de uma doença não transmissível antes dos 70 anos de idade era $44 \%$ maior em homens. Nos casos de suicídio também há predominância no sexo masculino e, a taxa global de 75\% mais altas em homens do que em mulheres em 2016. Mais uma vez se repete este fenômeno em acidentes de trânsito e mortalidade por homicídio. Este achado pode ser evidenciado pelo fato de as mulheres costumarem buscar mais os serviços de saúde e o atendimento médico que os homens. Além disso, a OMS destaca a importância do aumento da adesão aos serviços de saúde na atenção primária e a 
melhoria dos acessos e serviços de saúde, principalmente nas comunidades, o que é umas das principais metas da OMS para que se tenha, em 2023, uma cobertura universal de saúde.

Observou-se uma oscilação das taxas de letalidade e incidência da dengue nos anos de 2001 a 2018 no Estado de Mato Grosso, ora aumenta, ora diminui. Isso ocorre provavelmente devido a interação de diversos fatores, como as variáveis climáticas de pluviometria e temperatura, pois são fatores condicionantes para a variação de casos e produzem um efeito na dinâmica da dengue (Pelissari et al., 2016). Essas variações no período estudado estão diretamente relacionadas ao ambiente, circulação dos sorotipos, ações antrópicas, densidade populacional, alta densidade habitacional, urbanização não planejada e nível de infestação local pelo vetor da dengue, Aedes aegypti (Terra et al., 2017).

A faixa etária que mais adoeceu foram os adultos (20 a 59 anos) com uma taxa de incidência de 604,9/100 mil habitantes, correspondendo a 180.547 mil casos notificados, sendo que vieram a óbito 98 pessoas. Isso ocorreu também em Fortaleza no período de 2001 a 2002, em que todos os anos epidêmicos, proporcionalmente, notificaram-se maior incidência (Oliveira et al., 2018). Já a letalidade compromete mais os idosos em Mato Grosso. Isso se dá, hipoteticamente, em decorrência de diversos fatores, como por exemplo, ordem genética, estilo de vida e de exposição aos agentes ambientais, pois o envelhecimento é uma das fases da vida do ser humano e junto com ela chega inúmeras mudanças biossociomentais (biológico, mental e social), e com isso, os idosos podem ficar mais vulneráveis (Barbosa et al., 2020). A letalidade nos idosos também foi maior no Estado de Goiás no período de 2016-2018, correspondendo a 63,9\% (De Lima Oliveira et al., 2020).

A partir dos dados analisados, no período de 2001 a 2018, verificou-se uma superioridade do público feminino nas taxas de hospitalização por dengue $(51 \%)$, sendo que esse mesmo cenário se repete em um estudo realizado na região norte do país, em que o sexo feminino também apresentou 51,35\% das internações (De Moraes Dourado et al., 2020). O pico de taxa de internação hospitalar em todo o período estudado foi em 2010, sendo que o sexo feminino (178,3/100 mil habitantes) apresentou taxas superiores ao sexo masculino (156,4/100 mil habitantes). As mulheres são mais ativas, quando se trata de saúde, buscando com maior frequência as unidades de saúde.

Os homens tem maior dificuldade de buscar os serviços de saúde, devido à resistência em cuidar da saúde como forma preventiva, a falta de conhecimento, medo de descobrir doenças, ambiente feminino das unidades de saúde, fatores culturais que envolvem a construção da figura masculina, preconceito, machismo, jornada de trabalho sobrecarregada, fatores institucionais como a falta de recursos, disponibilidade de exames específicos, fatores culturais (machismo, sentimento de invulnerabilidade) e impaciência na espera por atendimento (Vieira et al., 2020). Segundo Brasil (2017), as internações hospitalares decorrentes da dengue no Brasil em 2013 foram duas vezes maiores onerosas para tratar os enfermos do que com a prevenção da dengue. Os gastos com o tratamento foram de $\mathrm{R}$ \$ 2,7 bilhões, enquanto que para a prevenção foi de $\mathrm{R} \$ 1,2$ bilhão. Além disso, no Estado de Goiás no período de 2016-2018 ultrapassou 4 milhões de reais (De Lima Oliveira et al., 2020).

\section{Conclusão}

A dengue revelou picos epidêmicos com altas taxas de incidência durante os 18 anos de estudo. O sexo feminino apresentou maior adoecimento e hospitalizações que o sexo masculino, porém o sexo masculino tem maior letalidade, tanto nos anos de pico, quanto em todo o período analisado. Os adultos adoecem mais que a demais faixas etárias, entretanto a letalidade é maior nos idosos, ou seja, apesar de se ter mais adultos acometidos pela enfermidade, os idosos acabam vindo a óbito, justamente pelas condições biológicas e clínicas das fases da vida humana. No Estado de Mato Grosso, a situação epidemiológica da dengue é preocupante, pois os registros de óbitos, incidência e internação hospitalar oscilam bastante 
Research, Society and Development, v. 10, n. 6, e32610615716, 2021

(CC BY 4.0) | ISSN 2525-3409 | DOI: http://dx.doi.org/10.33448/rsd-v10i6.15716

durante todo o período analisado, concluindo assim que, existem múltiplos fatores que influenciam nos picos epidêmicos da dengue. Há uma forte necessidade de investimentos satisfatórios em ações que possam combater essa doença, principalmente na educação ambiental. Essas medidas de controle devem ser de uma abordagem envolvendo vários setores: saúde, ambiente e educação, gerando assim maior impacto na população. Além disso, precisa haver investimento para poder fortalecer e aprimorar os serviços e sistemas de notificações. Dessa forma poderá reduzir a subnotificação e compreender melhor a dinâmica dessa enfermidade e de seu vetor no ambiente.

Apesar de ser complexa a problemática de controlar o vetor da dengue, é de extrema importância se modificar o atual cenário de constantes surtos e picos epidêmicos de dengue no estado e em todo o Brasil. Pois a cada ano milhares de pessoas vêm a óbitos devido a esta enfermidade, uma vez que é possível o combate ao vetor Aedes aegypti. Esta pesquisa foi importante para contribuir na elaboração de ações robusta a partir de uma abordagem de previsão de séries temporais da dinâmica da dengue. Dessa forma, pode-se fazer uma previsão do comportamento da dengue no Estado de Mato Grosso, evitando assim altas taxas de mortalidade e morbidade, diminuir os casos de hospitalizações e consequentemente corroborar com a diminuição de gastos com verbas hospitalares, além de poder planejar e executar ações que visem diminuir a proliferação do mosquito Aedes aegypti.

\section{Referências}

Barbosa, F. O., de Souza, D. M. T., Campos, G. O., de Almeida Carvalho, A. L. F., de Oliveira, B. M., Caminha, G. C., \& de Almeida, C. F. (2020). Perfil do envelhecimento entre usuários de em uma unidade de saúde em Campina Grande-PB: interface biopsicossocial. Brazilian Journal of Development, 6(7), $42392-42401$

Barbosa, I. R., Araújo, L. D. F., Carlota, F. C., Araújo, R. S., \& Maciel, I. J. (2012). Epidemiologia do dengue no Estado do Rio Grande do Norte, Brasil, 2000 a 2009. Epidemiologia e Serviços de Saúde, 21(1), 149-157.

Böhm, A. W., Costa, C. D. S., Neves, R. G., Flores, T. R., \& Nunes, B. P. (2016). Tendência da incidência de dengue no Brasil, 2002-2012. Epidemiologia e Serviços de Saúde, 25, 725-733.

de Lima Oliveira, D., Silva, Y. S., Naves, J. S., de Melo Júnior, G., Gonçalves, P. H. D., Silva, B. C. R., \& Silva, J. R. (2020). Custo das internações por dengue no Estado de Goiás, no período de 2016 a 2018. Brazilian Journal of Development, 6(5), 30695-30697.

de Oliveira, T. E. S., \& de Musis, C. R. (2014). Análise da flutuação das populações de aedes aegypti e aedes albopictus em uma escola de Cuiabá-MT. Revista Eletrônica em Gestão, Educação e Tecnologia Ambiental, 18(1), 178-186.

de Oliveira Medeiros, M., de Lima, L. J., \& Ferreira Filho, F. J. (2020). Morbidade hospitalar por dengue hemorrágica no Brasil (2010-2014).

de Morais Dourado, J., de Araújo, C. S., \& Godói, I. P. D. (2020). Hospitalizações, óbitos e gastos com dengue entre 2000 E 2015 na região norte do Brasil. Revista Brasileira Interdisciplinar de Saúde.

Fogaça, T. K., \& de Assis Mendonça, F. (2017). Dengue nas Américas: distribuição espacial e circulação viral (1995-2014). Hygeia-Revista Brasileira de Geografia Médica e da Saúde, 13(24), 175-188.

Fogaça, T. K., \& Mendonça, F. (2019). Distribuição espacial dos sorotipos de dengue e fluxos intermunicipais no Paraná. Raega-O Espaço Geográfico em Análise, 46(2), 101-115.

Governo do Estado de Mato Grosso, (2020). http://www.mt.gov.br/geografia\#: :text=Geografia\%20\%2D\%20mt.gov.br\&text=Mato\%20Grosso\%20tem\%20903.357\%2C908,do\%20Amazonas\%20e\%20d o\%20Par\%C3\%A1.\&text=O\%20local\%20 exato\%20foi\%20calculado,obelisco\%20da\%20C\%C3\%A2mara\%20dos\%20Vereadores.

Lucena, L. T. D., Aguiar, L. O., Bogoevich, A. C. A., Azevedo, F. S. D., Santos, A. C. P. D., Vale, D. B. A. P. D., \& Villalobos-Salcedo, J. M. (2011). Dengue na Amazônia: aspectos epidemiológicos no Estado de Rondônia, Brasil, de 1999 a 2010. Revista Pan-Amazônica de Saúde, 2(3), $19-25$.

Marchiori, E., Hochhegger, B., \& Zanetti, G. (2020). Manifestações pulmonares da dengue. Jornal Brasileiro de Pneumologia, 46(1).

Martins, M. M. F., Almeida, A. M. F. L., Fernandes, N. D. R., Silva, L. S., Lima, T. B. D., Orrico, A. D. S., \& Ribeiro Junior, H. L. (2015). Análise dos aspectos epidemiológicos da dengue na microrregião de saúde de Salvador, Bahia, no período de 2007 a 2014. Rev Espaço Saúde [Internet], 64-73.

Ministério da Saúde (2001). Fundação Nacional de Saúde. Dengue instruções para pessoal de combate ao vetor: manual de normas técnicas. Brasília. Ministério da Saúde. 
Research, Society and Development, v. 10, n. 6, e32610615716, 2021

(CC BY 4.0) | ISSN 2525-3409 | DOI: http://dx.doi.org/10.33448/rsd-v10i6.15716

Ministério da Saúde, (2017). Secretaria de Vigilância em Saúde. Coordenação-Geral de Desenvolvimento da Epidemiologia em Serviços. Guia de vigilância em saúde: volume 2. https://portalarquivos2.saude.gov.br/images/PDF/2017/outubro/16/Volume-Unico-2017.pdf.

Ministério da Saúde. (2019). Guia de vigilância em Saúde. Secretaria de Vigilância em Saúde. Coordenação-Geral de Desenvolvimento da Epidemiologia em Serviços. Guia de vigilância em saúde. Volume único, $3^{\text {a }}$ edição. https://bvsms.saude.gov.br/bvs/publicacoes/guia_vigilancia_saude_3ed.pdf.

Ministério da Saúde, (2020). Departamento de Informática do Sistema Único de Saúde (DATASUS). http://www2.datasus.gov.br/DATASUS/index.php.

Ministério da Saúde. (2006). Manual de normas e rotinas do Sistema de Informação de Agravos de Notificação. Brasília. Ministério da Saúde.

Ministério da Saúde, (2017). Secretaria de Vigilância em Saúde. Coordenação-Geral de Desenvolvimento da Epidemiologia em Serviços. Guia de vigilância em saúde: volume 2. https://portalarquivos2.saude.gov.br/images/PDF/2017/outubro/16/Volume-Unico-2017.pdf.

Ministério da Saúde. (2019). Guia de vigilância em Saúde. Secretaria de Vigilância em Saúde. Coordenação-Geral de Desenvolvimento da Epidemiologia em Serviços. Guia de vigilância em saúde. Volume único, $3^{a}$ edição. https://bvsms.saude.gov.br/bvs/publicacoes/guia_vigilancia_saude_3ed.pdf.

Ministério da Saúde, (2020). Departamento de Informática do Sistema Único de Saúde (DATASUS). http://www2.datasus.gov.br/DATASUS/index.php.

Ministério da Saúde. (2006). Programa Nacional de Controle da Dengue. Brasília. Ministério da Saúde. http://bvsms.saude.gov.br/bvs/politicas/programa_nacional_controle_dengue.pdf.

Ministério da Saúde, (2017). Secretaria de Vigilância em Saúde. Coordenação-Geral de Desenvolvimento da Epidemiologia em Serviços. Guia de vigilância em saúde: volume 2. https://portalarquivos2.saude.gov.br/images/PDF/2017/outubro/16/Volume-Unico-2017.pdf.

Ministério da Saúde. (2019). Guia de vigilância em Saúde. Secretaria de Vigilância em Saúde. Coordenação-Geral de Desenvolvimento da Epidemiologia em Serviços. Guia de vigilância em saúde. Volume único, $3^{a}$ edição. https://bvsms.saude.gov.br/bvs/publicacoes/guia_vigilancia_saude_3ed.pdf.

Ministério da Saúde, (2020). Departamento de Informática do Sistema Único de Saúde (DATASUS). http://www2.datasus.gov.br/DATASUS/index.php.

Oliveira, R. D. M. A. B., Araújo, F. M. D. C., \& Cavalcanti, L. P. D. G. (2018). Aspectos entomológicos e epidemiológicos das epidemias de dengue em Fortaleza, Ceará, 2001-2012. Epidemiologia e Serviços de Saúde, 27, e201704414.

Organização Mundial da Saúde, (2019). Folha informativa - Dengue e dengue grave. $2019 .<$ https://www.paho.org/bra/index.php?option=com_content\&view=article\&id=5963:folha-informativa-dengue-e-dengue-

rave\&Itemid=812\#: :text=A\%20dengue $\% 20 \% \mathrm{C} 3 \% \mathrm{~A} 9 \% 20$ uma $\% 20$ doen $\% \mathrm{C} 3 \% \mathrm{~A} 7 \mathrm{a}$,propor\%C3\%A7\%C3\%A3o\%2C\%20da\%20esp \%C3\%A9cie\%20Aedes

\%20albopictus>

Organização Pan-americana, (2019). de Saúdehttps://www.paho.org/bra/index.php?option=com_content\&view=article\&id=5963:folha-informativa-dengue-edengue-

grave\&Itemid=812\#: :text=Estimativas $\% 20$ recentes\%20indicam\%20390\%20milh\%C3\%B5es,com\%20qualquer\%20gravidade\%20da\%20doen\%C3\%A7a.

Pan American Health Organization. Dengue. 2017. http://www.paho.org/hq/index.php?option=com_topics\&view=article\&id=1\&Itemid=40734

Secretaria Estadual de Saúde de Mato Grosso, (2016). Vigilância em Saúde. Relatório de infestação do Aedes aegypti. http://www.saude.mt.gov.br/suvsa.

Secretaria Estadual de Saúde de Mato Grosso, (2019). Vigilância em Saúde. Vetores Transmissores de doenças. http://www.saude.mt.gov.br/suvsa.

Teich, V., Arinelli, R., \& Fahham, L. (2017). Aedes aegypti e sociedade: o impacto econômico das arboviroses no Brasil. JBES: Brazilian Journal of Health Economics/Jornal Brasileiro de Economia da Saúde, 9(3).

Terra, M. R., DA Silva, R. S., Pereira, M. G. N., \& Lima, A. F. (2017). Aedes aegypti e as arbovíroses emergentes no Brasil. Revista Uningá Review, 30(3).

Toyama, D., Santino, M. B. D. C., \& Fushita, A. T. Qualidade da água e doenças de veiculação hídrica: o caso dos municípios de barra bonita e Igaraçu do Tietê-SP. V SIGA Ciência (Simpósio Científico de Gestão Ambiental), 1.

Vieira, U. A., de Oliveira Araujo, M., de Oliveira Araujo, B., \& do Nascimento Paixão, G. P. (2020). Percepção dos enfermeiros sore a (não) procura dos homens por Atenção Primária à Saúde. Revista de Saúde Coletiva da UEFS, 10(1), 58-66.

Villar, J. E., \& Gonçalves, B. C. M. Dengue: origem, espécie e tratamento. II Semana da Farmácia UniFATEA 18, 19 e 20 de outubro de 2016, 6.

World Health Organization. (1997). Dengue haemorrhagic fever: diagnosis, treatment, prevention and control. World Health Organization.

World Health Organization. (2019). World health statistics overview 2019: monitoring health for the SDGs, sustainable development goals (No. WHO/DAD/2019.1). World Health Organization. 